\title{
Oxaliplatin and Bolus-Modulated 5-Fluorouracil as a Second-Line Treatment for Advanced Pancreatic Cancer: Can Bolus Regimens Replace FOLFOX When Considered for Second Line?
}

\author{
A. Azmy, ${ }^{1}$ S. Abdelwahab, ${ }^{2}$ and M. Yassen ${ }^{2}$ \\ ${ }^{1}$ GIT and Gynecological Oncology Unit, Ain Shams University, Cairo 11539, Egypt \\ ${ }^{2}$ Department of Clinical Oncology, Ain Shams University, Cairo 11539, Egypt
}

Correspondence should be addressed to A. Azmy; alyazmy68@hotmail.com

Received 1 November 2012; Accepted 20 December 2012

Academic Editors: G. Ferrandina and P. Karakitsos

Copyright (C) 2013 A. Azmy et al. This is an open access article distributed under the Creative Commons Attribution License, which permits unrestricted use, distribution, and reproduction in any medium, provided the original work is properly cited.

\begin{abstract}
Objective. Comparing activity of 2 regimens combining oxaliplatin to bolus modulated fluorouracil as second line treatment in advanced pancreatic adenocarcinoma pretreated with gemcitabine-containing schedule. Methods. Forty eight patients with advanced pancreatic adenocarcinoma were randomly assigned to receive either FU $500 \mathrm{mg} / \mathrm{m}^{2}$ IV bolus weekly $\times 6$ weeks plus leucovorin $500 \mathrm{mg} / \mathrm{m}^{2}$ IV weekly for 6 weeks during each 8-week cycle plus oxaliplatin $85 \mathrm{mg} / \mathrm{m}^{2}$ IV on weeks 1,3 , and 5 of each 8 -week (FLOX) OR receive weekly intravenous infusions of oxaliplatin $40 \mathrm{mg} / \mathrm{m}^{2}, 5$-FU $500 \mathrm{mg} / \mathrm{m}^{2}$, and leucovorin $250 \mathrm{mg} / \mathrm{m}^{2}(3$ weeks on, 1 week off). Results. Non progression(PR+SD) was found in $33.5 \%$ for first regimen and $29 \%$ for second regimen, and $37.5 \%$ had clinical benefit (FLOX regimen) compared to 50\% in 3-weeks regimen. The median TTP was 3.9,4 months respectively. Median OS was 8, 9 months for both regimens. Only one case in 3-weeks arm suffered from grade IV diarrhea. Two cases $>$ grade 2 neutropenia were observed; one in each treatment groups. Grade 3 anemia was recorded in 3 patients ( 2 in FLOX arm, one in 3-weeks arm). Conclusions. Both regimens showed encouraging efficacy, acceptable toxicity, and clinical benefit.
\end{abstract}

\section{Introduction}

Due to the fact that the majority of pancreatic cancers are unresectable upon diagnosis, curative intent is rarely a goal of treatment, rather increasing survival, time to progression, and quality of life are more realistic goals. Without treatment, median survival for patients with an advanced stage of disease ranges from 3 to 4 months, whereas in patients receiving chemotherapy with singleagent gemcitabine, median survival times between 4.9 and 7.2 months have been reported in randomized phase III studies [1]. Gemcitabine has been the solo player in the field of pancreatic cancer, treatment after replacing 5-FU since 1997, and is still regarded as one standard of care for the first-line systemic chemotherapeutic treatment of patients with advanced pancreatic cancer worldwide. So far, only two randomized phase III trials have demonstrated a significant prolongation of survival with the use of gemcitabine-based combination therapy with either erlotinib or capecitabine [2]. Eventually, progression will occur and the real challenge will be how to treat a patient with advanced pancreatic cancer failing to respond or progressing after gemcitabine. There is no evidence-based treatment recommendation for these patients. The National Comprehensive Cancer Network guidelines for pancreatic adenocarcinoma currently recommend second-line chemotherapeutic treatment after gemcitabine failure in selected patients using, for example, single-agent capecitabine or a combination therapy of fluorouracil, leucovorin and oxaliplatin (FOLFOX-) like regimen [3]. To date, there is no large randomized trial confirming the survival advantages of second-line chemotherapy over best supportive care, yet the preliminary results from a small 
randomized German study comparing BSC alone versus 5FU, folinic acid, and oxaliplatin plus BSC after gemcitabine failure showed a prolongation of median survival by approximately 2.6 months with the use of chemotherapy ( 2.3 versus 4.9 months) [4]. These data were supported by a Japanese study that reported a median survival time of approximately 1.9 months after failure of first-line gemcitabine in 74 patients with pancreatic cancer (of whom $97 \%$ received no secondline treatment) [5]. Many protocols containing oxaliplatin, 5FU, and leucovorin, as FOLFOX, FLOX, and 3-week bolus 5FU plus leucovorin and oxaliplatin, are known. Preclinical data suggested that the 5-FU plus oxaliplatin combination is more cytotoxic when 5-FU is given as a short exposure [6], which gives a rationale for exploring the toxicity and efficacy of such protocols in advanced pancreatic cancer. In the current study, we conducted a randomized trial to compare two protocols; FLOX and the 3-weeek bolus protocol regarding toxicity, response rate, and time to progression as primary end points, then overall survival as secondary endpoints.

\section{Patients and Methods}

Patients with advanced unresectable or metastatic pancreatic adenocarcinoma were enrolled under the following Eligibility criteria.

Inclusion Criteria. (I) Patients with histologically or cytologically proven locally advanced or metastatic pancreatic adenocarcinoma, (II) with at least 1 bidimensionally measurable lesion (World Health Organization (WHO) criteria); (III) Eastern Cooperative Oncology Group (ECOG) PS of 1-2; (IV) tumor progression after first line gemcitabine (whether gemcitabine pretreated or gemcitabine resistance); (V) absence of severe uncontrolled cardiovascular, metabolic, infectious, or neurological diseases; (VI) adequate bone marrow reserve (neutrophil count $>1.5 \times 10^{9} / \mathrm{L}$, platelet count $>100.000 / \mathrm{mm}^{3}$ and $\mathrm{Hb}>10 \mathrm{~g} / \mathrm{dL}$ ); (VII) adequate liver function (serum bilirubin $<1.5 \mathrm{mg} / \mathrm{dL}$, serum transaminases $<2 \mathrm{x}$ the upper limit of normal); (VIII) adequate renal function (serum creatinine $<1.5 \mathrm{mg} / \mathrm{dL}$ ); (IX) and age between 18 and 75 years. All participating patients were required to give written informed consent, and ethical approval from MOC committee was obtained before the start of the whole procedure.

\section{Exclusion Criteria}

(i) Histologic types other than adenocarcinoma.

(ii) Neuropathy $\geq$ CTCAE grade 1.

(iii) Ototoxicity $>$ CTCAE grade 2.

(iv) Serious, active comorbidity, including any of the following: unstable angina and/or NYHA class IIIV congestive heart failure requiring hospitalization within the past 12 months, transmural myocardial infarction within the past 12 months, acute bacterial or fungal infection requiring IV antibiotics, chronic obstructive pulmonary disease exacerbation or other respiratory illness requiring hospitalization or precluding study therapy, hepatic insufficiency resulting in clinical jaundice and/or coagulation defects, active gastrointestinal (GI) ulcers, GI bleeding, inflammatory bowel disease, or GI obstruction, Inadequately controlled hypertension, defined as systolic BP > $150 \mathrm{~mm} \mathrm{Hg}$ and/or diastolic $\mathrm{BP}>90 \mathrm{~mm} \mathrm{Hg}$ on antihypertensive medications, serious cardiac arrhythmia on medication (wellcontrolled atrial fibrillation on medication allowed), and history of hypertensive crisis or hypertensive encephalopathy.

2.1. Pretreatment Evaluation. All patients were subjected to staging procedures consisted of medical history, physical examination, echocardiography, serum chemistry panel, complete blood picture, CEA, and CA 19-9. Extent of disease was determined by chest X-rays, computed tomography and/or nuclear magnetic resonance, and endoscopy as needed. Patients underwent followup examinations until death.

2.2. Randomization Procedures. Patients were randomly assigned to one of the treatment regimens (block randomization at 4), where 24 patients were enrolled for each treatment group.

\subsection{Treatment}

2.3.1. FLOX Regimen. Oxaliplatin $85 \mathrm{mg} / \mathrm{m}^{2}$ was administered as a 2-hour infusion before $\mathrm{LV}$ and $\mathrm{FU}$ on days 1,15 , and 29 of the treatment cycle. LV $500 \mathrm{mg} / \mathrm{m}^{2}$ was administered as a 2-hour intravenous infusion weekly for 6 consecutive weeks (on days 1, 8, 15, 22, 29, and 36 of the treatment cycle), followed by a 2 -week rest period. FU $500 \mathrm{mg} / \mathrm{m}^{2}$ was administered as an intravenous bolus 1 hour after the LV infusion was begun and was administered weekly for 6 weeks (on days 1, 8, 15, 22, 29, and 36 of the treatment cycle), followed by a 2 -week rest period.

2.3.2. 3-Week Bolus Regimen. 2-hour intravenous infusion of oxaliplatin $40 \mathrm{mg} / \mathrm{m}^{2}$ was followed by bolus leucovorin $250 \mathrm{mg} / \mathrm{m}^{2}$ and bolus 5 -FU $500 \mathrm{mg} / \mathrm{m}^{2}$. Each course consisted of weekly administrations for 3 consecutive weeks followed by a week of rest. Therapy continued until disease progression, unacceptable toxicity, patient's refusal, or a maximum of 6 courses.

All patients received intravenous dexamethasone $8 \mathrm{mg}$, Ondansitrone $8 \mathrm{mg}$ as antiemetic prophylaxis. Therapy was withheld in case of a platelet count of less than $100.000 / \mathrm{mm}^{3}$ or a neutrophil count of less than $1.500 / \mathrm{mm}^{3}$ or for bilirubin greater than 1.5 times the upper reference level (URL) or transaminases greater than 3 times the URL. During the entire study period, patients received full supportive care to control pain or other symptoms, with careful recording of the treatment. 
2.4. Toxicity. Adverse events were graded according to the National Cancer Institute common toxicity. Oxaliplatin was reduced in the event of persistent paresthesia/dysesthesia between cycles or with pain lasting for $>7$ days according to staff physician's decision. When paresthesia/dysesthesia with either pain or functional impairment persisted between cycles, Oxaliplatin was discontinued.

2.5. Evaluation and Statistical Methods. Measurable disease response was assessed by RECIST criteria [7]. Partial response (PR), stable disease (SD), and progressive disease (PD) were determined according to these criteria. The sum of PR and SD was reported as disease control rate (DCR). OS was estimated from the date of first treatment to the date of death or the last followup. Clinical benefit assessment was based on patients and physician-reported improvement of cancer-related symptoms and/or stabilization of improvement of PS. The TTP was calculated from the first treatment infusion to the first objective evidence of disease progression assessed by CT scan measurements or early death or date of clinical deterioration and patient not assessable for response. All patients with at least 1 chemotherapy administration were assessed for toxicity. Efficacy assessments were performed on patients who received at least 1 course of therapy. TTP and OS since the start of treatment were estimated on an intentto-treat basis and analyzed according to the Kaplan-Meier method. Comparison between survival curves was done through $\log$ rank test to estimate $P$ value utilizing GraphPad prism version 5 software. The required number of patients for this phase II study was determined according to a Jehan phase II optimal design [8] for a goal of $20 \%$ true clinical benefit; with $\alpha$ - and $\beta$-error probability of 0.05 and 0.20 , respectively, an accrual of 24 patients assessable for response was planned.

\section{Results}

Forty-eight patients with unresectable or metastatic pancreatic cancer pretreated with gemcitabine (including gemcitabine resistance or gemcitabine pretreated) in Ain Shams University Hospitals were included along the period between October 2008 and September 2011.

The patients' characteristics encountered in the current study were outlined in Table 1.

Median age for both groups was 56 years and 54 years, respectively. Sixteen males out of total twenty-four cases were encountered in FLOX arm compared to 17 in 3-week bolus arm. Thirty patients in both groups received prior Gemcitabine as a single agent.

3.1. Toxicities. Grade 3 or 4 toxicities experienced by at least $5 \%$ of patients according to treatment arm are summarized in Table 2.

Only one case in 3-week arm suffered from grade IV diarrhea. Two cases of neutropenia exceeding grade 2 (but no febrile neutropenia) were observed; one in each treatment groups. Grade 3 anemia was recorded in 3 patients ( 2 in FLOX
TABLE 1: Patients' characteristics.

\begin{tabular}{|c|c|c|c|}
\hline Characteristics & FLOX arm & 3-week arm & $P$ value \\
\hline \multicolumn{4}{|l|}{ Age } \\
\hline Median & 56 & 54 & .45 \\
\hline Range & $44-69$ & $41-68$ & \\
\hline \multicolumn{4}{|l|}{ Gender } \\
\hline Male & $16(67 \%)$ & $17(71 \%)$ & .49 \\
\hline Female & $8(33 \%)$ & $7(29 \%)$ & .35 \\
\hline \multicolumn{4}{|l|}{ Disease at presentation } \\
\hline Locally advanced & $15(63 \%)$ & $14(58 \%)$ & .44 \\
\hline Metastatic & $9(37 \%)$ & $10(42 \%)$ & .23 \\
\hline \multicolumn{4}{|l|}{ Site of metastases } \\
\hline Liver & $5(21 \%)$ & $6(25 \%)$ & .48 \\
\hline Lung & $1(4 \%)$ & $1(4 \%)$ & - \\
\hline $\mathrm{LN}$ & $2(8 \%)$ & $2(8 \%)$ & - \\
\hline Peritoneal & $1(4 \%)$ & $1(4 \%)$ & - \\
\hline others & - & & \\
\hline \multicolumn{4}{|l|}{ Previous surgery } \\
\hline Palliative & $5(21 \%)$ & $4(17 \%)$ & .3 \\
\hline Radical & $1(4 \%)$ & $1(4 \%)$ & - \\
\hline None & & & \\
\hline \multicolumn{4}{|l|}{ Prior chemotherapy } \\
\hline Gemcitabine single agent & $14(58 \%)$ & $16(67 \%)$ & .23 \\
\hline $\mathrm{Gem}+5 \mathrm{FU}$ & $8(34 \%)$ & $6(25 \%)$ & .17 \\
\hline Gem + Platinol & $2(8 \%)$ & $2(8 \%)$ & - \\
\hline \multicolumn{4}{|l|}{ Response to prior therapy } \\
\hline Partial response & - & - & \\
\hline Stable disease & $2(8 \%)$ & $3(12.5 \%)$ & .12 \\
\hline Progression & $22(92 \%)$ & $21(87.5 \%)$ & .27 \\
\hline \multicolumn{4}{|l|}{ Presented symptoms } \\
\hline Pain & $8(34 \%)$ & $8(34 \%)$ & - \\
\hline Weight loss & $20(84 \%)$ & $20(84 \%)$ & - \\
\hline
\end{tabular}

arm, one in 3-week arm). Most nonhematological side effects were less than grade 3.

3.2. Efficacy. No complete response was registered among all assessable 48 patients throughout the study duration for FLOX regimen, three patients (12.5\%) had partial response, five patients $(21 \%)$ had stable disease, and three out of 8 patients with pain at presentation $(37.5 \%)$ had clinical benefit. The median time to progression was 3.9 months (95\% CI, 2-4.6) (range: 1.5-5.5). Median survival time was 8 months (95\% CI, 4.0-12).

For 3-week regimen, two patients (8\%) had partial response, five patients (21\%) had stable disease, and four out of 8 patients with pain at presentation (50\%) had clinical benefit as shown in Table 3. The median time to progression was 4 months (95\% CI, 1.8-5) (range: 1.2-6). Median survival time was 9 months (95\% CI, 3.5-13) as shown in Figures 1(a) and $1(\mathrm{~b})$.

There was no statistical significance in progression-free survival between the 2 regimens $(P$ value by log rank test $=$ 
TABLE 2: Patients with grade 3 or 4 toxicity by the NCI Common Toxicity Criteria version 3.0.

\begin{tabular}{|c|c|c|c|c|c|}
\hline \multirow{2}{*}{ Characteristics } & \multicolumn{2}{|c|}{ FLOX arm } & \multicolumn{2}{|c|}{ 3-week arm } & \multirow[t]{2}{*}{$P$ value } \\
\hline & Grade III & Grade IV & Grade III & Grade IV & \\
\hline Diarrhea & $4(16 \%)$ & $1(4 \%)$ & $4(16 \%)$ & $1(4 \%)$ & .11 \\
\hline Dehydration & $2(8 \%)$ & - & $1(4 \%)$ & - & .09 \\
\hline Nausea & $1(4 \%)$ & - & $2(8 \%)$ & - & .101 \\
\hline Vomiting & $3(12 \%)$ & - & $1(4 \%)$ & - & .07 \\
\hline Stomatitis & $1(4 \%)$ & - & $1(4 \%)$ & - & - \\
\hline \multicolumn{6}{|l|}{ Hematological } \\
\hline Neutropenia & $1(4 \%)$ & & $1(4 \%)$ & - & - \\
\hline Anaemia & $2(8 \%)$ & & $1(4 \%)$ & - & .089 \\
\hline Thrombocytopenia & - & - & - & & \\
\hline Neurosensory & $1(4 \%)$ & - & $1(4 \%)$ & - & - \\
\hline Thrombosis and embolism & $1(4 \%)$ & - & $1(4 \%)$ & - & - \\
\hline \multicolumn{6}{|l|}{ Liver } \\
\hline Elevated transaminases & $2(8 \%)$ & - & $3(4 \%)$ & - & .21 \\
\hline
\end{tabular}

TABLE 3: Summarizing efficacy results of the 2 regimens.

\begin{tabular}{lccc}
\hline & FLOX arm & 3-week arm & $P$ value \\
\hline Partial responses & $3(12.5 \%)$ & $2(16 \%)$ & .23 \\
Stable disease & $5(21 \%)$ & $5(21 \%)$ & .5 \\
Clinical benefit & $3 / 8(37.5 \%)$ & $4 / 8(50 \%)$ & .19 \\
\hline
\end{tabular}

.4619), and so was the situation in overall survival ( $P$-value by $\log$ rank test $=.5248)$.

3.3. Cost Comparison. Although it was not planned as a target for the current study, yet it was an interesting issue to compare cost of chemotherapy per patient for every 8 weeks of treatment for each regimen. For FLOX regimen this cost was approximately 1200 USD versus 1400 USD for the 3 -weeks regimen (due to mainly the amount of discarded oxaliplatin in every injection time that was more in the second regimen) as in Table 4.

\section{Discussion}

Advanced pancreatic cancer remains a rapidly lethal cancer, with a median survival of 6 months with currently approved therapies [8]. The role of second-line chemotherapy after failure of first-line therapy in such cases is not well established, but a theoretical possibility exists in which salvage chemotherapy after the failure of first-line treatment may influence the survival. For the scale of patients with good performance status, progressing after first-line gemcitabine therapy, NCCN recommends fluoropyrimidinebased chemotherapy [9]. But for time being there is still a debate to treat or best supportive care? A phase III trial after failure of first-line gemcitabine compared BSC plus with biweekly oxaliplatin combined with weekly 5-FU as 24 hours infusion plus leucovorin, versus BSC alone [4]. After the first 46 patients out of 165 planned, the BSC arm had to be closed because BSC alone was no longer accepted by participating centers, with a possible survival benefit for second-line chemotherapy: 21 weeks (95\% CI; .7; 23.3) versus 10 weeks (95\% CI; $7.7 ; 12.3$ ). So a second question is as the following: what is the best option of treatment? Adding oxaliplatin to continuous infusion fluoropyrimidine as a second-line salvage therapy for this category has been investigated in some phase II trials [10-12]. In a series of unselected patients the FOLFOX4 regimen yielded a 14\% PR rate with $38 \%$ of patients showing SD for a DCR of $57 \%$. Median duration of PR was 5.2 months, while median time to progression and overall survival was 4 and 6.7 months, respectively [10], but all the regimens of continuous infusion necessitate either hospitalization or pump application with their financial load upon health care system. This was the rationale to investigate regimens including oxaliplatin and bolus fluorouracil, with the theoretical premise of being as active as continuous infusion regimens, as well simpler in administration, less in cost, and better in toxicity profile. In the current study, two regimens of oxaliplatin and bolus fluorouracil have been investigated, FLOX regimen that was used in metastatic colorectal cancer with adequate efficacy and acceptable toxicity profile [12]. The current study revealed nonprogression ( $\mathrm{PR}+\mathrm{SD}$ ) in $33.5 \%$ for first regimen and $29 \%$ for second regimen, and $37.5 \%$ had clinical benefit (FLOX regimen) compared to $50 \%$ in 3-week regimen. The median time to progression was 3.9 months and 4 months, respectively. Median survival time was 8 months and 9 months for both regimens, respectively, with no statistically significant difference in progression-free or overall survival. Regarding toxicity, only one case in 3-week arm suffered from grade IV diarrhea. Two cases of neutropenia exceeding grade 2 (but no febrile neutropenia) were observed; one in each treatment group. Grade 3 anemia was recorded in 3 patients ( 2 in FLOX arm, one in 3-week arm). Most nonhematological side effects were less than grade 3 .

So it is the time for the 3rd question; are these results comparable to those of FOLFOX regimens (infusion fluorouracil)? Gebbia et al., 2007, carried out a retrospective study including 42 patients who received standard FOLFOX4 


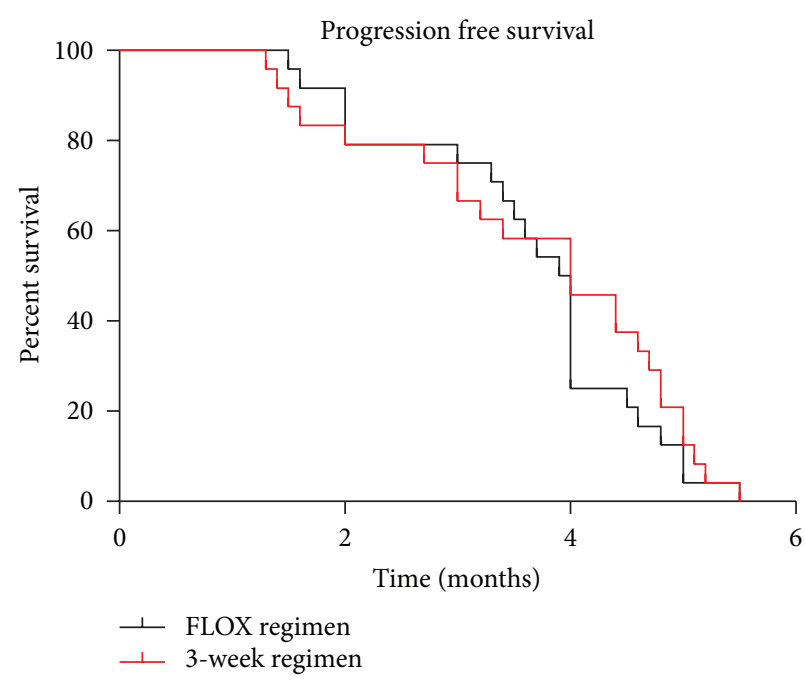

(a)

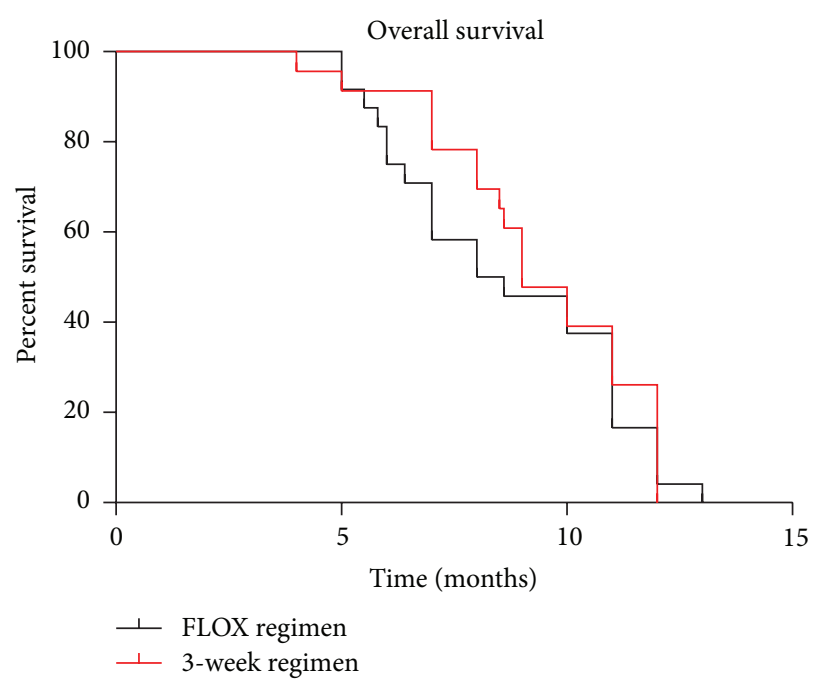

(b)

Figure 1: (a) Progression-free survival; Kaplan-meier curves of both treatment regimens. (b) Overall survival; Kaplan-Meier curves of both treatment regimens.

TABLE 4: Cost comparison of the 2 regimens.

\begin{tabular}{lccc}
\hline & FLOX arm & 3-week arm & $P$ value \\
\hline Cost/patient/8 weeks & 1200 USD & 1400 USD & .013 \\
\hline
\end{tabular}

regimen biweekly until progression or unacceptable toxicity. The study revealed six partial responses (14\%) and 16 stabilizations (38\%) were recorded for a tumor growth control rate of $57 \%$. The median time to progression (TtP) was 4 months (range 1-7 months), and median overall survival (OS) was 6.7 months (range 2-9 months). A stabilization of performance status (PS) and a subjective improvement of cancer-related symptoms was recorded in 27 patients [13]. The good nonprogression rate in this study may be attributed to the high percentage of responding patients in this study to the first line therapy (50\%) compared to $12 \%$ in our study.

Tsavaris et al., 2005, in a prospective Phase II study evaluated a second-line combination regimen of oxaliplatin together with leucovorin-modulated 5-FU in 30 patients and revealed an encouraging response rate of $23 \%$ with a corresponding disease-control rate of 53\%. Median overall survival was 5.8 months in this patient population [14]. These data correlate well with the retrospective analysis from Italy, which found a response rate of $14 \%$ together with a diseasecontrol rate of $52 \%$ with the use of a FOLFOX- 4 regimen in gemcitabine-pretreated patients [14].

Another phase II trial of oxaliplatin plus capecitabine in a series of 41 patients reported a PR in one case and SD in eight patients with a median OS of 5.8 months, and a 6-month and 1 -year survival rate of $48 \%$ and $22 \%$, respectively [9]. Toxicity was, however, significant. Preliminary results of another trial of OXP/5-FU in a series of 23 patients have shown an OS of 4 months [15].

Novarino et al., 2009, in a study on 23 gemcitabine pretreated patients with advanced pancreatic cancer revealed no objective response in all 17 assessable patients and 4 patients had stable disease, whereas 13 had tumor progression. Median duration of stable disease was 14 weeks. Median time to progression of disease (TTP) was 11.6 weeks. Seven patients experienced grade 3-to- 4 toxicity. The regimen was associated with $36 \%$ clinical benefit [16].

In conclusion, combining oxaliplatin to bolus fluorouracil (either in FLOX or 3-week regimens) as a second line in gemcitabine pretreated patients with advanced or metastatic pancreatic adenocarcinoma showed encouraging efficacy, acceptable toxicity, and some clinical benefit specially when palliation or good quality of life is a target keeping in mind the simplicity in administration, the no need for hospitalization, and the less financial load specially with FLOX. Further studies with large number of patients investigating the efficacy and tolerability of such bolus regimens in gemcitabinepretreated pancreatic cancer patients are warranted.

\section{References}

[1] H. S. Hochster, D. G. Haller, A. De Gramont et al., "Consensus report of the International Society of Gastrointestinal Oncology on therapeutic progress in advanced pancreatic cancer," Cancer, vol. 107 , no. 4 , pp. 676-685, 2006.

[2] D. Cunningham, I. Chau, D. D. Stocken et al., "Phase III randomized comparison of gemcitabine versus gemcitabine plus capecitabine in patients with advanced pancreatic cancer," Journal of Clinical Oncology, vol. 27, no. 33, pp. 5513-5518, 2009.

[3] M. A. Tempero, S. Behrman, E. Ben-Josef et al., "Pancreatic adenocarcinoma. Clinical practice guidelines in oncology," Journal of the National Comprehensive Cancer Network, vol. 3, no. 5, pp. 598-626, 2005, http://www.nccn.org/index.asp.

[4] U. Pelzer, I. Schwaner, J. Stieler et al., "Best supportive care (BSC) versus oxaliplatin, folinic acid and 5-fluorouracil (OFF) plus BSC in patients for second-line advanced pancreatic cancer: a phase III-study from the German CONKO-study 
group," European Journal of Cancer, vol. 47, no. 11, pp. 1676-1681, 2011.

[5] K. Nakachi, J. Furuse, H. Ishii, E. I. Suzuki, and M. Yoshino, "Prognostic factors in patients with gemcitabine-refractory pancreatic cancer," Japanese Journal of Clinical Oncology, vol. 37, no. 2, pp. 114-120, 2007.

[6] J. L. Fischel, M. C. Etienne, P. Formento, and G. Milano, "Search for the optimal schedule for the oxaliplatin/5-fluorouracil association modulated or not by folinic acid: preclinical data," Clinical Cancer Research, vol. 4, no. 10, pp. 2529-2535, 1998.

[7] P. Therasse, S. G. Arbuck, E. A. Eisenhauer et al., "New guidelines to evaluate the response to treatment in solid tumors: European Organization for Research and Treatment of Cancer, National Cancer Institute of the United States, National Cancer Institute of Canada," Journal of the National Cancer Institute, vol. 92, pp. 205-216, 2000.

[8] T. T. Chen and T. H. Ng, "Optimal flexible designs in phase II clinical trials," StatMed, vol. 17, no. 20, pp. 2301-2312, 1998.

[9] H. A. Burris III, M. J. Moore, J. Andersen et al., "Improvements in survival and clinical benefit with gemcitabine as firstline therapy for patients with advanced pancreas cancer: a randomized trial," Journal of Clinical Oncology, vol. 15, pp. 24032413, 1997.

[10] "NCCN Clinical practice guidelines in oncology (NCCN GuidelinesTM) pancreatic adenocarcinoma version 2," 2012, http://www.nccn.org/professionals/physician_gls/f_guidelines .asp.

[11] E. Gebbia, E. Maiello, F. Giuliani et al., "Second-line chemotherapy in advanced pancreatic carcinoma: a multicenter survey of the Gruppo Oncologico Italia Meridionale on the activity and safety of the FOLFOX4 regimen in clinical practice," Annals of Oncology, vol. 18, no. 6, pp. vil24-vil27, 2007.

[12] H. Oettle, U. Pelzer, J. Stieler et al., "Oxaliplatin/folinic acid/5fluorouracil [24h] (OFF) plus best supportive care versus best supportive care alone (BSC) in second-line therapy of gemcitabine-refractory advanced pancreatic cancer (CONKO 003)," J Clin Oncol, vol. 23, no. 16S, article 4031, 2005.

[13] U. Pelzer, C. Hempel, J. Stieler et al., "Oxaliplatin (OXA) in combination with high dose 5 -FU $(24 \mathrm{~h}) /$ folinic acid (FA) as salvage therapy in patients with gemzar-refractory advanced pancreatic cancer," Proceedings of American Society of Clinical Oncology, vol. 21, article 172, 2002.

[14] J. - Philip Kuebler, H. Samuel Wieand, J. Michael O'Connell, E. Roy Smith, H. Linda Colangelo et al., "Oxaliplatin combined with weekly bolus fluorouracil and leucovorin as surgical adjuvant chemotherapy for stage II and III colon cancer: results from NSABP C-07," JCO, vol. 25, no. 16, pp. 2199-2204, 2007.

[15] V. Gebbia, E. Maiello, F. Giuliani et al., "Second-line chemotherapy in advanced pancreatic carcinoma: a multicenter survey of the Gruppo Oncologico Italia Meridionale on the activity and safety of the FOLFOX4 regimen in clinical practice," Annals of Oncology, vol. 18, no. 6, pp. vi124-vi127, 2007.

[16] N. Tsavaris, C. Kosmas, H. Skopelitis et al., "Second-line treatment with oxaliplatin, leucovorin and 5-fluorouracil in gemcitabine-pretreated advanced pancreatic cancer: a phase II study," Investigational New Drugs, vol. 23, no. 4, pp. 369-375, 2005. 


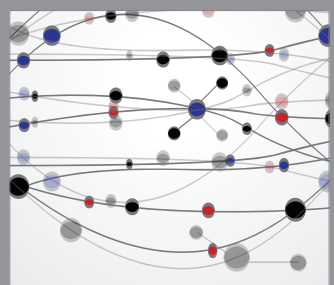

The Scientific World Journal
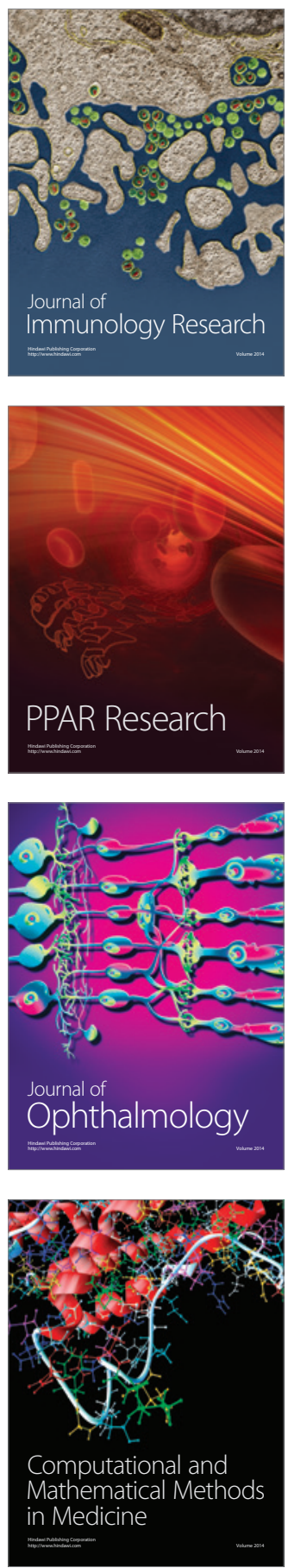

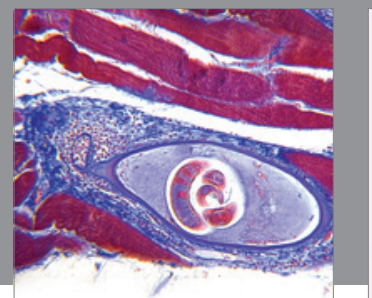

Gastroenterology

Research and Practice
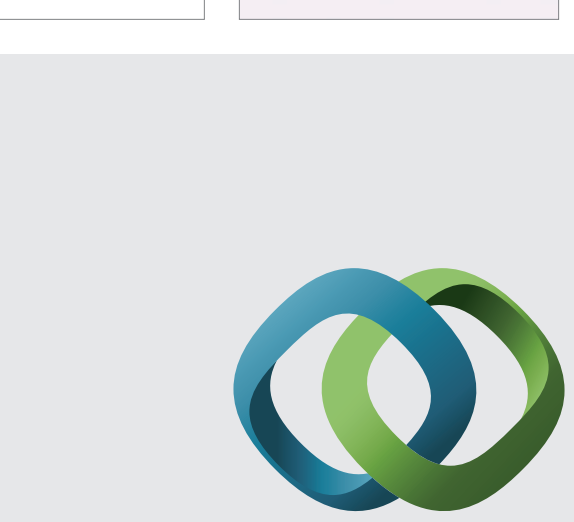

\section{Hindawi}

Submit your manuscripts at

http://www.hindawi.com
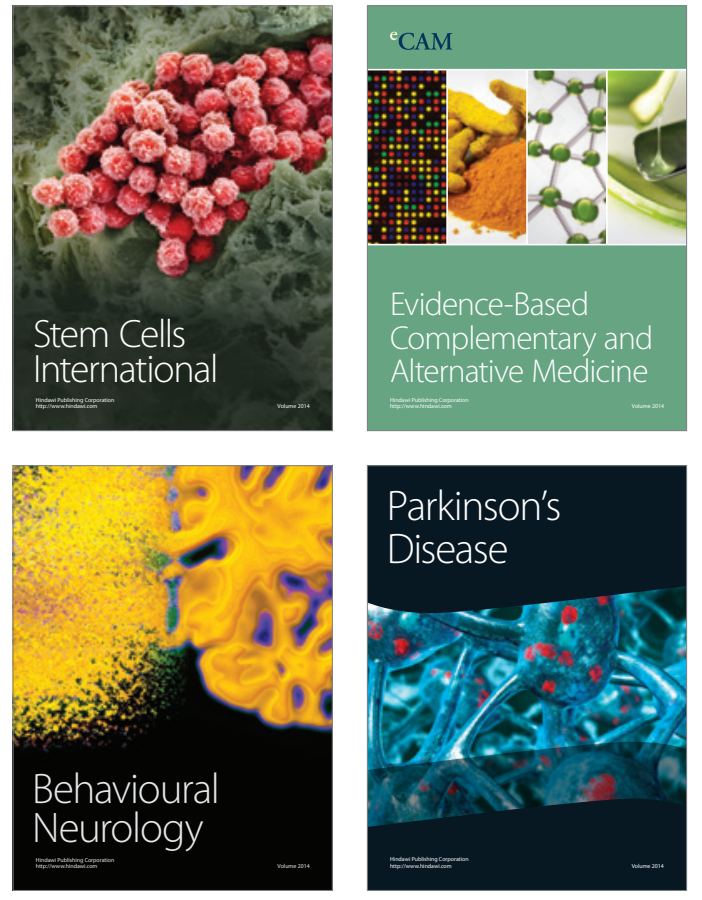
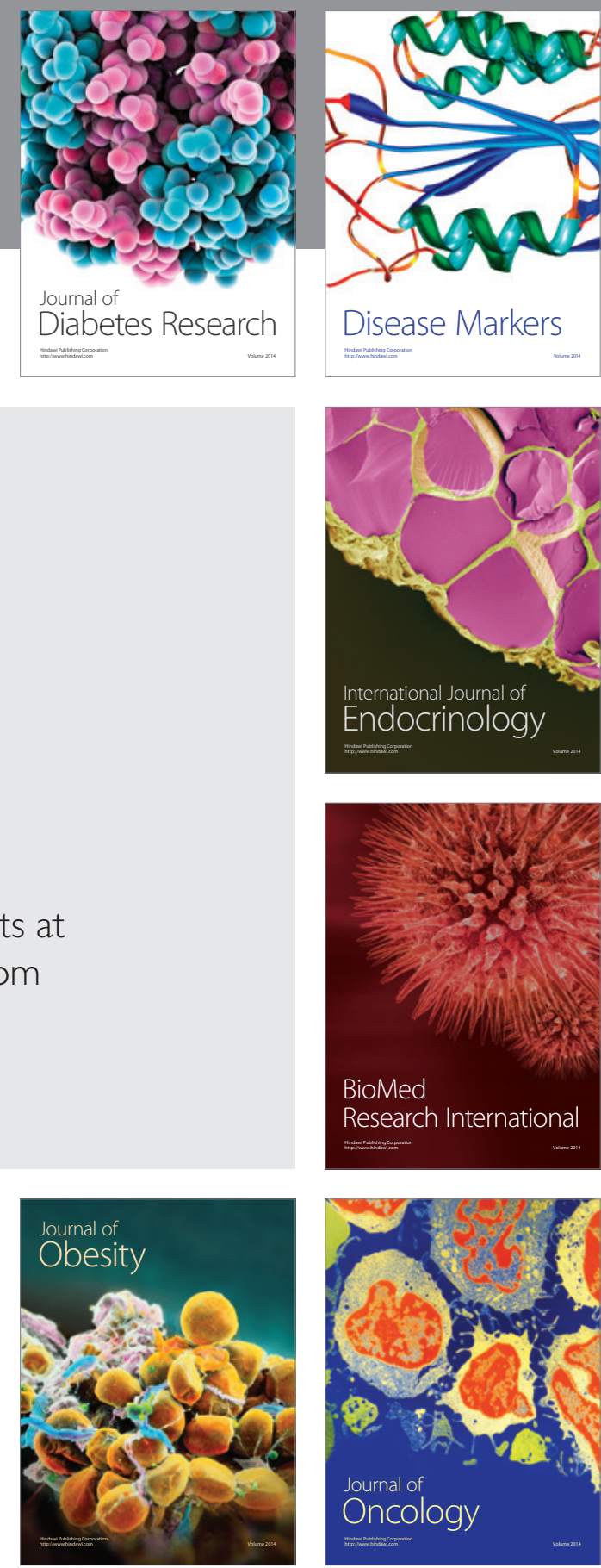

Disease Markers
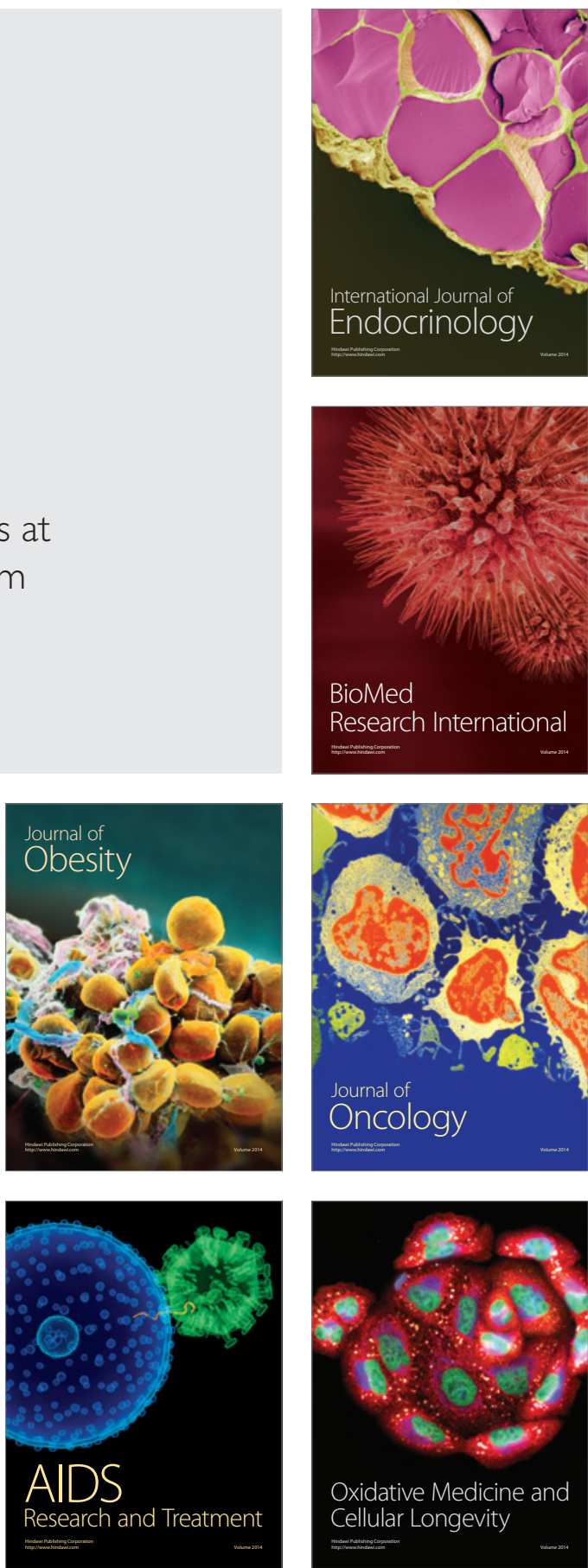